\title{
AN APPROACH TO CONTROL INTER CELLULAR INTERFERENCE USING LOAD MATRIX IN MULTI CARRIER MOBILE COMMUNICATION SYSTEMS
}

\author{
Srinivas Karedla ${ }^{1}$, Santhi Rani ${ }^{2}$ \\ ${ }^{1}$ Assistant Professor, Department of Electronics and Communications, GITAM University, Visakhapatnam, India \\ ${ }^{2}$ Professor, Department of Electronics and Communications, DMSSVH College of Engineering, Machilipatnam, India
}

\begin{abstract}
This paper deals with reduction of inter cellular interference in Multi-carrier communication systems. In the past, Load Matrix $(L M)$ is proposed to allocate power to different users in a network based upon Signal to noise plus interference ratio (SNIR) so as to reduce inter cellular interference and is observed for single carrier systems. In Multi carrier systems the SNIR is affected distinctly in each carrier thus a single SNIR for power allocation is not optimal. In this paper, to obtain the optimization of power allocation in Multi-Carrier system, Load Matrix coding with bifurcated SNIR (LM-BFSNIR) is proposed. Using this approach it is observed that inter cellular interference is reduced better when compared to a single carrier system evaluated over a $3 G P P-L T E$ standard.
\end{abstract}

Keywords-Power allocation, Inter cellular interference, Multi-Carrier mobile Communication system.

\section{INTRODUCTION}

The objective of wireless communication systems is to offer secured transmission by maximizing the data rate. To achieve this aim modern technologies in wireless communications are introduced in which multi-Carrier communication is thriving at a rapid rate. Typically, for higher data rate transmission extra band width is required. Yet, because of spectral limitations, it is usually absurd or very expensive to rise bandwidth. The alternative solution is to use multiple transmit and receive antennas for deployment of available spectrum. Usage of multiple transmit and receive antennas forms a multi-carrier communication system. Advantages of multi-carrier over single carrier are fine quality of service, less fraction of dropped calls and more capacity of network . Capacity of a network can be improved if multiple receive and transmit antennas are used as multi carrier channels [1]. In [2] it is shown thatmulti-carrier systems do better than the single carrier with Rayleigh flat fading . Capacity of a multi carrier system depends upon the number of receive and transmit antennas. In Multi-carrier [3] by partitioning the entire channel ( spectrum ) to many narrow parallel sub channels so that width of symbol increases and thus reducing the inter symbol interference generated by the multipath. In [4] it is shown that co-channel interference is decreased by using adaptive resource scheme in multi-carrier system. LM [5] is a resource allocation scheme that allocates the power to the available base stations and users based on the SNIR to minimize inter cellular interference in turn reduce bit error rate as outlined in the section I and is evaluated only for single carrier systems .In multi carrier systems the SNIR is affected distinctly in each carrier and hence by using a diverse approach ie LM with bifurcated SNIR (LM-
BFSNIR) the bit error rate can be decreased better in multi carrier than single carrier in reducing the inter cellular interference better.In this paper the proposed LM-BFSNIR approach is applied to multi carrier over 3GPP LTE standards .

\section{SYSTEM OUTLINE}

Multi-Carrier technology has procured much significance and users consideration because of its applications in mobile communication, digital television, wireless LAN's and MAN's. The channel capacity of multi-carrier system is directly proportional to transmitter and receiver arrays, which have been increased remarkably in multi carrier system. Second, system performance and accuracy have been enhanced in multi-carrier system as a result it provides a spatial variety in which each transmitting signal is detected by the whole detector array. This also minimizes the ISI (inter symbol interference) effect and impact of channel fading since each signal is determined based on ' $\mathrm{N}$ ' detected results i.e. spatial diversity offers ' $\mathrm{N}$ ' independent replicas of transmitted signal. Third advantage of multi carrier system is that the array gain can be increased, which implies that SNR gain can be obtained by increasing directivity i.e. by focusing energy in required direction.

A Multi carrier system is designed for test evaluation with four transmit and $\varphi(\varphi=4)$ receive antennas is developed. Transmitted data block can be defined as, $\left\{h_{n}[r, o]: o=\right.$ $1,2 \ldots$.$\} These signals are two different signals$ $\left\{x_{2(m-1)+n[r, o]}: o=1,2 \ldots ; r=1,2 \ldots\right\}$ for $\mathrm{m}=1$ and 2 respectively through two space-time encoders. The $\mathrm{m}^{\text {th }}$ transmitting antenna signal is modulated by $x_{m}[r, o]$ at the $\mathrm{o}^{\text {th }}$ carrier of the $\mathrm{r}^{\text {th }}$ data block. The received signal is a 
superposition of four distorted transmitted Signals at each receiving antenna, given by

$$
v_{n}[r, o]=\sum_{m=1}^{4} L_{m, n}[r, o] x_{m}[r, o]+a_{n}[r, o]
$$

For $n=1 \ldots s$ where $a_{n}[r, o]$ is the additive complex Gaussian noise at the $\mathrm{n}^{\text {th }}$ receive antenna, and is assumed to be zero-mean with variance $\sigma_{n}^{2}$ uncorrelated for different $r$ 's, o's and n's. The Channel frequency response corresponding to the $\mathrm{m}^{\text {th }}$ transmit and the $\mathrm{n}^{\text {th }}$ receive antenna for the o ${ }^{\text {th }}$ tone at time $\mathrm{r}$ is $L_{m, n}[r, o]$

The vector form representation of the Multi-carrier system relation shown in (1) can be expressed as

$$
\begin{gathered}
v_{n}[r, o]=\sum_{i=1}^{4} L_{1}[r, o] \\
a_{n}[r, o]
\end{gathered} \quad x_{1}[r, o]+L_{2}[r, o] x_{2}[r, o]+
$$

Where

$$
L_{m}[r, o]=\left(\begin{array}{cc}
L_{2 m-1,1}[r, o] & L_{i m, 1}[r, o] \\
\vdots & \vdots \\
L_{2 m-1, t}[r, o] & L_{2 m, t}[r, o]
\end{array}\right)
$$

Multiple power levels are owed to the transmitting signals to transmit over the channel. The effective utilization of resources is due to the best possible utilization of transmitting power. To achieve effective allocation of resources a "resource allocation" approach has been designed which has been outlined in the following section.

\section{LOAD MATRIX [ 5] APPROACH}

Load Matrix (LM) approach has the characteristic of jointly supervising the interference within a cell while allocating radio resources to users and to circumvent Rise over thermal noise (RoT) outage by taking into consideration the inter cell interference information. The management of inter cell interference is the main difficulty for resource allocation in multi-cell system. LM is a centralized scheduler which provides radio resources to all dynamic users in the network by using a database containing the load part of all dynamic users in the network.

The critical problem in the uplink scheduler is to assign proper transmission rate and time to all dynamic users, to result in utmost utilization of radio resources across the network while rewarding the QoS necessities of all the users. The significant issue in the resource allocation is the users transmit power. The subsequent Limitations are to be satisfied for a network of $\mathrm{N}$ users and $\mathrm{M}$ cells/base stations . Limitation 1: This states that for each dynamic user $i$ in the network the transmitted power $u_{n}$ must be in an acceptable region defined as

$$
0 \leq u_{n} \leq u_{\max }, n \in\{1,2 \ldots N\}
$$

Where $u_{\max }$ is the maximum user power.

Limitation 2: A definite threshold level for all $M$ base stations must be maintained and the total received power at base station must be kept below this threshold. In the network RoT can be used to be a sign of the interference constraints.

$$
R o T_{o} \leq R o T_{\text {target }}, o \in\{1, \ldots . M\}
$$

Where $R o T_{o}$ is the total band received power, preset target value to maintain uplink interference level at the base station o $\left(\mathrm{BS}_{\mathrm{o}}\right)$ over thermal noise. For a network containing $\mathrm{M}$ dynamic users the RoT of cells can be estimated by using RoT $j$, which can be written as

$$
\operatorname{RoT}_{o}=\left(\frac{M^{*}+\sum_{n=0}^{N} u_{n} G_{n, o}}{M^{*}}\right)
$$

Limitation 3 :The signal to noise plus interference ratio necessary at the $o^{\text {th }}$ serving base station if rate ' $p$ ' is being assign to the user to attain a given frame error rate is SNIR target, $p$. For each user, depending on its channel form and speed, each rate $\mathrm{p}$ has a least required $S N I R$ called $S N I R$ target, $p$. This constraint satisfies only by considering SNIR target $k$ as SNIR.

$$
S N I R \geq S N I R_{\text {target }, p} n \in\{1, \ldots N\} ; p \in\{1, \ldots P\}
$$

LM is nothing except a centralized scheduler assigns radio resources to all the $\mathrm{N}$ users and $\mathrm{M}$ cells in the network, $L M n, o$ is the load factor contribution by user $\mathrm{n}$ at $B S o$ defined as

$$
L M_{n, o}=\frac{u_{n} G_{n o}}{M^{*}+\sum_{n=1}^{N} u_{n} G_{n o}}
$$

Where Gno is the channel gain from user $\mathrm{n}$ to $\mathrm{BS}_{\mathrm{o}}$ averaged over scheduling period, $M^{*}$ is the thermal noise and $u_{n}$ is the transmitted power. The LM n,o values stored in column 'o' of LM database, RoT of cell 'o' can be written as

$$
R o T_{o}=\frac{1}{1-\sum_{n=1}^{N} L M_{n, o}}
$$

SNIR $n, o$ can be written as

$$
S N I R_{n, o}=\frac{u_{n} G_{n o}}{M^{*} R o T_{o}-u_{n} G_{n o}}
$$

The necessary transmitted power for user $\mathrm{n}$ at rate $\mathrm{p}$ is,

$$
u_{n, o}=\frac{\left[M^{*} R o T_{o}\right]\left[S N I R_{\text {target }, o}\right]}{G_{n o}\left[1+S N I R_{\text {target }, o}\right]}
$$

If above all Limitations are satisfied then only power $u_{i, k}$ is acceptable and user $\mathrm{n}$ will be scheduled for transmission. After that LM elements are updated and RoT is calculated for each cell using [10]. The performance of the LM scheduling has the best RoT over other algorithms because this scheduler significantly reduces the probability of the RoT exceeding its target. The RoT is computed over a single carrier system. The Load matrix approach considered in the previous section is able to allocate the power efficiently for a single cell users based on SNIR and RoT concepts, considering only signal to noise ratio for power allocation for single carrier communication. 


\section{LM - BFSNIR APPROACH}

In Multi-carrier communication system the Signal to interference ratio is affected distinctly in each carrier hence a single SNIR for allocation is not optimal. Also, since due to usage of orthogonally modulated signals for transmission over narrow band channels the interference between them is decreased.

Hence SIR which is distinct in each channels is almost maximized ,for the optimization of power allocation the total SIR in Multi carrier system is sum of SIR's in individual channels which greater than the SNIR obtained in single carrier system. This can be mathematically expressed as

$$
S N I R=\left(\frac{1}{S N R}+\frac{1}{S I R}\right)^{-1}
$$

In equation (12), the SNIR used in single carrier systems is bifurcated in terms of SNR and SIR

Where $S N R=\frac{u_{n}}{\sigma_{b n}^{2}}$ denotes the background signal-to-noise ratio. SIR is the signal-to-interference ratio evaluated for in every narrow channel by doing so the (12) can be rewritten as shown

$$
B F S N I R=\left(\frac{1}{S N R}+\frac{1}{\sum_{i=0}^{\varphi} S I R_{i}}\right)^{-1}
$$

Where BFSNIR is called as bifurcated SNIR evaluated for multi-carrier system designed with four transmit and receive antennas(ie $\varphi=4$ ), substituting the above equation (13) for the conventional LM approach, the proposed SNIR $n, o$ get modified as

$$
B F S N I R=\frac{u_{n} G_{n o}}{M^{*} R o T_{o}-u_{n} G_{n o}}+\frac{u_{t}}{\sum_{n=1}^{M} u_{n}}
$$

The required transmitted power for user $i$ at rate $p$ is then defined as,

$$
u_{n, p}=\frac{M^{*} R_{0} T_{o} G_{n o}}{G_{n o}} \frac{\text { BFSNIR }_{\text {target }, p}}{1+B F S N I R_{\text {target }, p}}
$$

The allocable power to each user with rate $\mathrm{p}$ is then defined as;

$$
P_{\text {alloc }}=u_{n, p} \text { if BFSNIR }<u_{n, p}
$$

Else

user will be in queue and scheduled for transmission next Transmission time interval(TTI)

\section{SIMULATION RESULTS}

For the simulation of the planned work a 3GPP-LTE standard wireless communication model is taken. The considered communication parameter for the evaluation is given as in Table 1;
The proposed work is evaluated using MATLAB and the simulated results are as follows

Table 1

\begin{tabular}{|l|l|l|}
\hline S.No & Parameter & Description \\
\hline 1 & Category & 1 \\
\hline 2 & $\begin{array}{l}\text { Uplink_data } \\
\text { rate(UL-DR_peak })\end{array}$ & 5 Mbps \\
\hline 3 & $\begin{array}{l}\text { Down link data rate } \\
\text { (DL-DR_peak) }\end{array}$ & $\begin{array}{l}\text { 10Mbps(used } \\
5 \mathrm{mbps})\end{array}$ \\
\hline 4 & RF Bandwidth & 20 MHz(CC) \\
\hline 5 & Modulation & $\begin{array}{l}\text { Uplink-16 QAM } \\
\text { Downlink- } \\
16 \mathrm{QAM}\end{array}$ \\
\hline 6 & Architecture & $\begin{array}{l}\text { Uplink-MIMO } \\
\text { 4x4 (OFDM) }\end{array}$ \\
\hline 7 & Channel condition & Urban Micro \\
\hline 8 & $\begin{array}{l}\text { Channel } \\
\text { Factor Delay }\end{array}$ & $\begin{array}{l}\text { 1.627e-8sec } \\
\text { Random }\end{array}$ \\
\hline
\end{tabular}




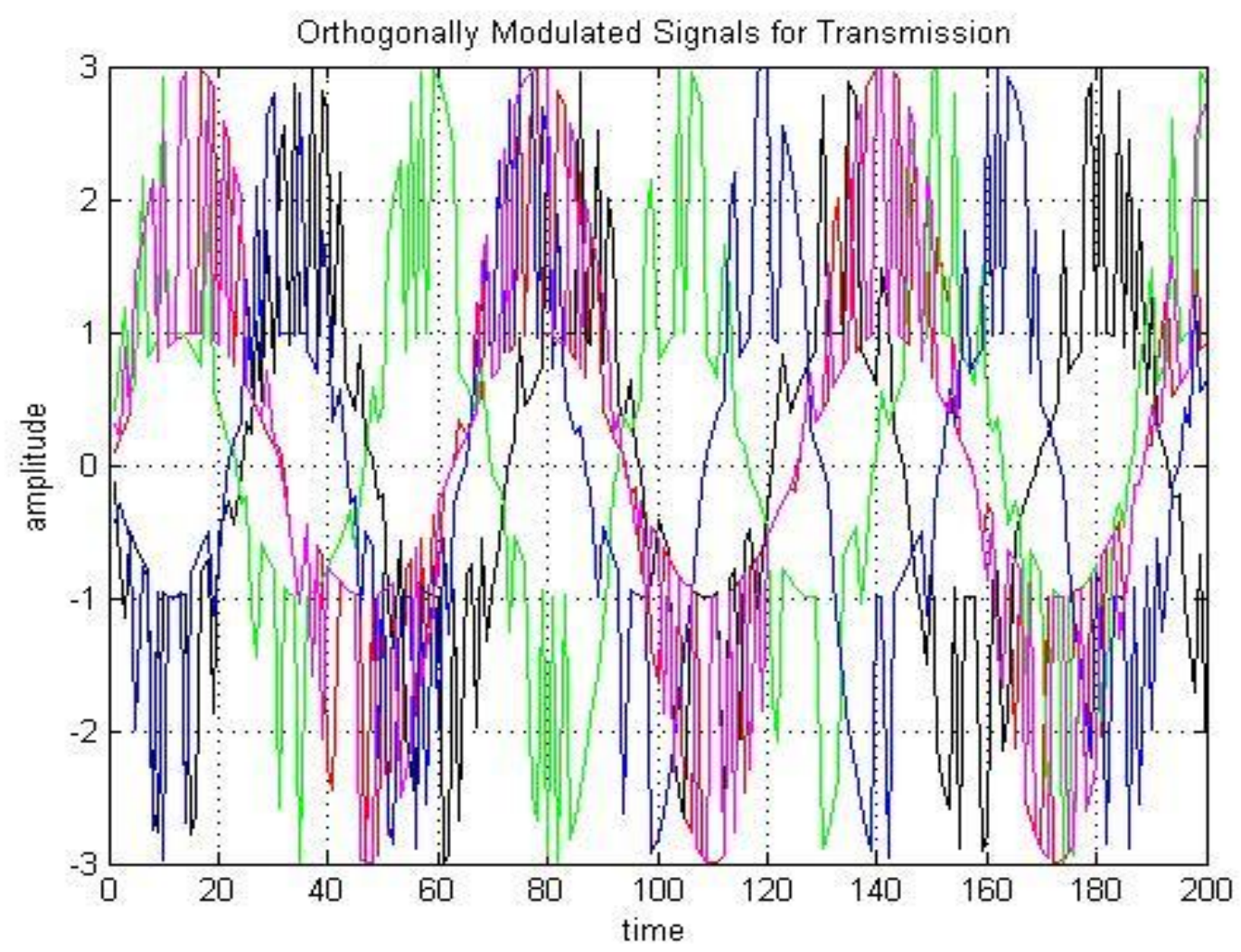

Fig 1: Plot of orthogonally modulated signals used for transmission

The above figure shows the orthogonally modulated signals for transmission

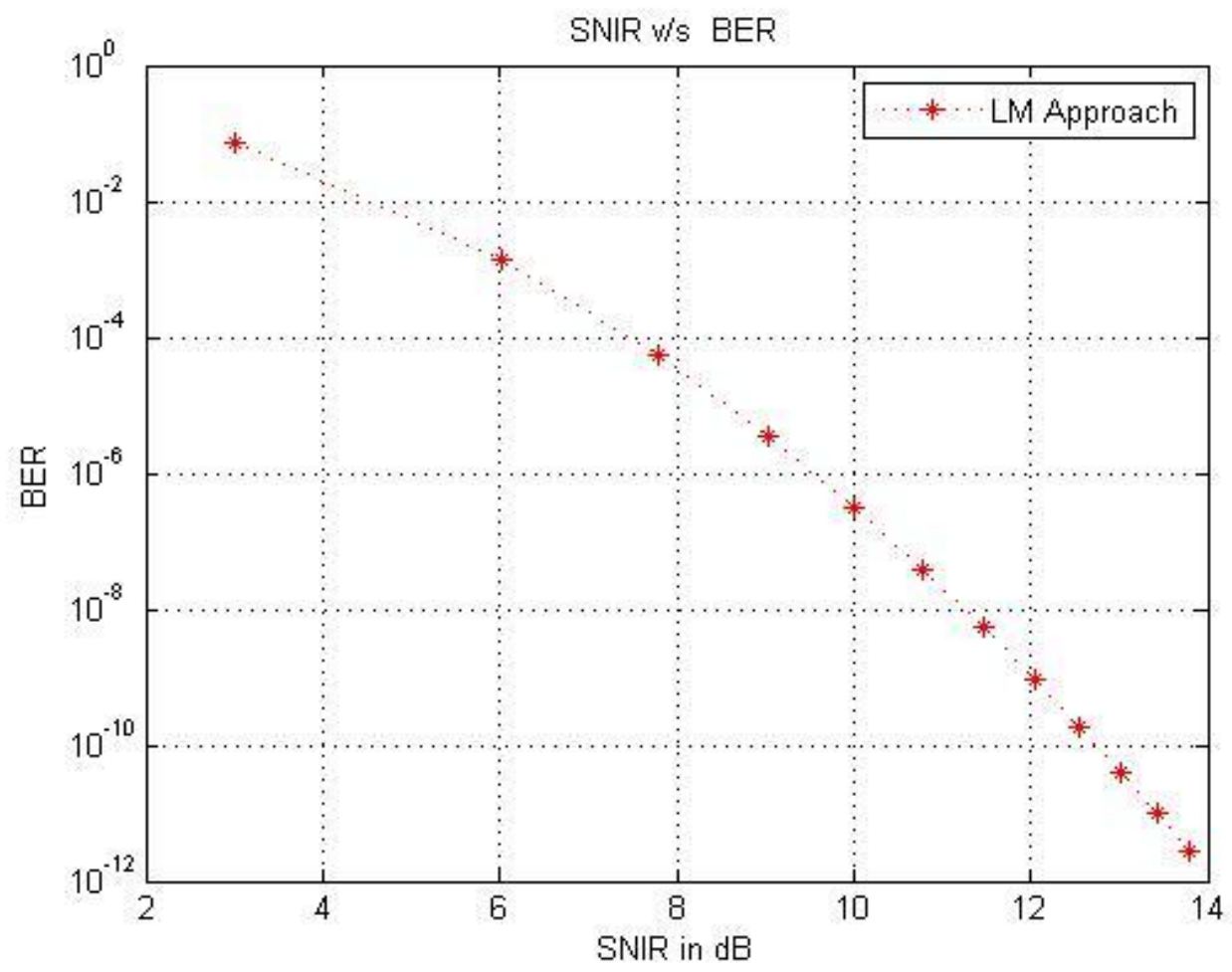

Fig 2: SNIR vs BER plot for the multi Carrier system using LM coding

The figure above shows the variation of Bit Error Rate (BER) for different values of SNIR evaluated over multi carrier mobile communication systems . 


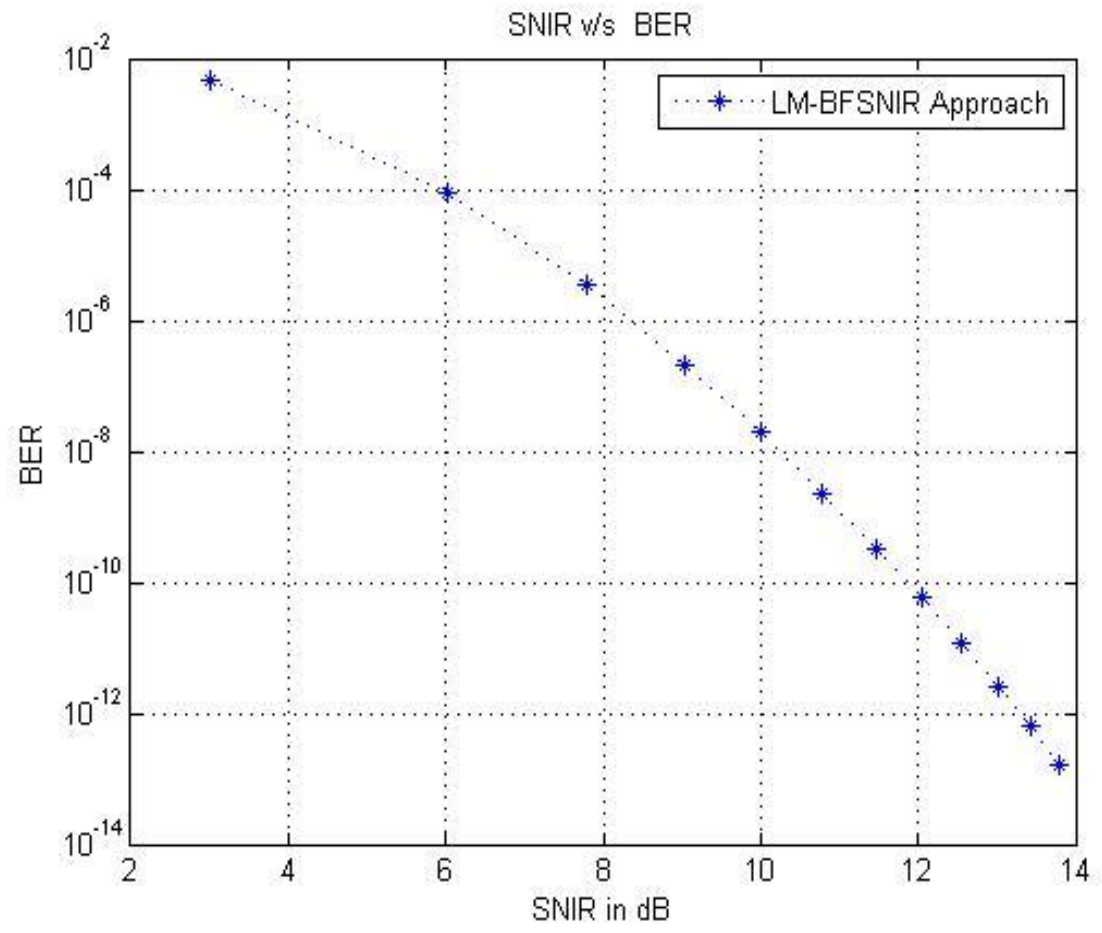

Fig 3: SNIR vs BER plot for the multi Carrier system using LM-BFSNIR coding

The result outlines the obtained BER over variable SNIR at different data rate.By observing the figures 2 and 3 it can be seen that the BER decreases noticeably by using LM-BF SNIR coding than the conventional LM coding. For example for a SNIR of $13 \mathrm{db}$ the BER decreases from $10^{-11}$ to $10^{-13}$ using LM-BFSNIR compared to LM coding .

\section{CONCLUSION}

In this paper, LM coding is evaluated for multi-carrier mobile communication system . In past work Load matrix approach is applied for single carrier system and the power is allocated to the users by considering SNIR.By considering the LM-BFSNIR approach in multi-carrier system it is observed that the inter cellular interference is reduced better than the single carrier systems and in turn reduces the bit error rate .

\section{REFERENCES}

[1]. R.R.Chen and Y.Lin. "Optimal power Control for Multiple Access Channel and Average Power Constraints.".In proceedings of IEEE International Conference on Wireless Networks, Communication and Mobile Computing, pp 147-1411.2005.

[2]. J.H Winters "On the Capacity of radio communication systems with diversity in a Rayleigh fading environment. "IEEE J. Select. Areas Communication,vol.SA-5,pp 871878,june 1987.

[3]. G. J. Foschini, "Layered space-time architecture for wireless communication in a fading environment when using multi-element antennas," Bell Labs Tech. J., pp. 4159, Autumn 1996.
[4]. G. J. Foschini, G. D. Golden, R. A. Valenzuela, and P. W. Wolniansky, "Simplified processing for high spectral efficiency wireless communication employing multielement arrays," IEEE J. Select. Areas Communication., vol. 17, pp. 1841-1852, Nov. 1999.

[5]. Mohammad Abaii, Yajian Liu, and Rahim Tafazolli, "An Efficient Resource Allocation Strategy forFuture Wireless Cellular Systems", IEEE Transactions on Wireless Communications, Vol. 7, No. 8, August 2008. 